\title{
Analysis of Surface Parametrizations for Modern Photometric Stereo Modeling
}

\author{
Roberto Mecca $^{a}$, Emanuele Rodolà $^{b}$, and Daniel Cremers ${ }^{b}$ \\ ${ }^{a}$ Department of Mathematics-CIRAM, University of Bologna, Bologna (Italy) - \\ roberto.mecca@unibo.it; \\ ${ }^{b}$ Computer Vision Group, Technische Universität München, Garching (Germany), \\ [rodola,cremers]@in.tum.de
}

\begin{abstract}
Tridimensional shape recovery based on Photometric Stereo (PS) recently received a strong improvement due to new mathematical models based on partial differential irradiance equation ratios. ${ }^{1}$ This modern approach to PS faces more realistic physical effects among which light attenuation and radial light propagation from a point light source. Since the approximation of the surface is performed with single step method, accurate reconstruction is prevented by sensitiveness to noise. In this paper we analyse a well-known parametrization ${ }^{2}$ of the tridimensional surface extending it on any auxiliary convex projection functions. Experiments on synthetic data show preliminary results where more accurate reconstruction can be achieved using more suitable parametrization specially in case of noisy input images.
\end{abstract}

Keywords: Photometric stereo, surface parametrization, partial differential equations, image ratio.

\section{INTRODUCTION}

The three-dimensional representation of the surrounding world through accurate shape reconstructions has increased its importance together with the computational power of current computers capable to process enough data for shape recovery in a reasonable amount of time. The process of reconstructing shapes from shading information has been studied from the seminal paper about the Shape from Shading (SfS) problem ${ }^{3}$ (single image) and enforced by the extension to the Photometric Stereo (PS) technique ${ }^{4}$ (multiple images). Relating to the SfS, several approaches have been presented ${ }^{5,6}$ attempting to prove its well-posedness. In this framework, the way to parametrize surface and light beam is crucial. For example, Prados and Faugeras ${ }^{2}$ derived a specific pinhole parametrization for the surface in the perspective scenario showing that their particular formulation made the SfS problem well-posed. Following, after few years Breuß et al. ${ }^{7}$ showed that it was actually not completely well-posed.

On the other hand, PS is one of the monocular techniques for three-dimensional reconstruction providing very accurate shape recovery. The main drawback of this technique is the very limited capability to work in realistic scenarios. For example, instead of considering shaped light sources which spread light depending on their geometry and positions, the light sources are usually considered placed far away from the observed object in order to simplify the incident light as a uniform beam, without considering consequently light attenuation. In order to model simple physical conditions, most of the literature in the PS field solves standard mathematical problems by minimizing energy functionals ${ }^{8,9}$ or computing pseudo-inverse matrices. ${ }^{4,10}$ In other words, a suitable parametrization of the problem can actually make the difference on formulating a PS model in order to face realistic scenarios and provide suitable formulation yielding to more appropriate mathematical problems.

In this direction, Partial Differential Equations (PDEs) are becoming an important mathematical tool for modeling PS under realistic physical assumptions.

Unlike very common procedures that need to approximate the normal field ${ }^{4}$ and afterwards the depth, ${ }^{11-14}$ recent PDE methods based on image ratios allow direct computation of the surface. For example, Chandracker et al. ${ }^{15,16}$ consider more general irradiance equations with unknown light sources, and compute the photometric invariants describing the surface through its isocontours. ${ }^{17}$ However, the shape reconstruction process requires additional initial or boundary information. Mecca et al. ${ }^{18,19}$ use specific irradiance equations for diffuse surfaces, 


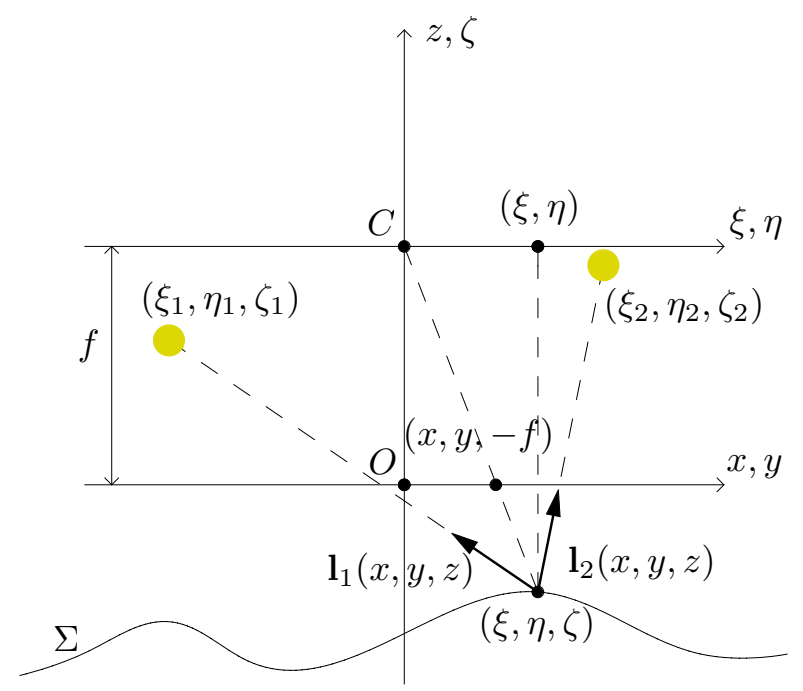

Figure 1. A schematic section of the derivation of the perspective view geometry. In the perspective world (i.e., the image coordinate system given by $O x y z)$ the light reflected at image point $(x, y)$ comes form the real point $(\xi, \eta, \zeta)$ of the surface.

and the uniqueness of solution is proved by characteristic strip expansion and assuming known light information (i.e., direction or position).

Related to this last approach, another step towards more realistic PS was undertaken in ${ }^{1}$ where the close-range problem for a scene lit by point-light sources is approached with quasi-linear PDEs parametrizing different types of realistic light attenuations. An iterative numerical scheme based on the fast-marching method is used in order to approximate the shape. In this framework, the importance of using an appropriate parametrization becomes clear. In this paper, we explore more in detail how different parametrizations of a surface $\Sigma$, modeled as a function $\mathbf{M}: \bar{\Omega} \rightarrow \mathbb{R}^{3}$ (where $\bar{\Omega}=\Omega \cup \partial \Omega$ is the image domain), can affect the reconstruction in terms of shape accuracy.

Our contributions can be summarized as follows:

- We generalize the parametrization presented in ${ }^{2}$;

- We derive the model presented in ${ }^{1}$ with respect to the previous type of parametrization;

- We show how these different parametrizations can influence the accuracy of the shape recovery, due to the numerical approximation of the respective quasi-linear PDEs.

\section{MODEL DERIVATION}

We recall how the PS problem is formulated in ${ }^{1}$ starting by the well-known irradiance equation for Lambertian surfaces, given by the following inner product:

$$
I_{i}(x, y)=\rho(x, y) a_{i}(x, y)\left[\overline{\mathbf{n}}(x, y) \cdot \overline{\mathbf{l}}_{i}(x, y)\right]
$$

where $\mathbf{n}$ is the outgoing normal to the surface, $I_{i}, \rho: \bar{\Omega} \rightarrow[0,1]$ are the image function taken under the $i$-th light source and the unknown albedo respectively. Here we indicate as ${ }^{*}$ a normalized vector.

With the aim to model realistic attenuation effects, we consider the function derived in ${ }^{1}$ :

$$
a_{i}(x, y)=\left(\frac{l_{i}^{3}(x, y)}{\mathbf{l}_{i}(x, y)}\right)^{\mu}
$$

where $\mu \geq 0$ describes the amount of the attenuation. 
Since the point light source is assumed to be fixed at point $P_{i}=\left(\xi_{i}, \eta_{i}, \zeta_{i}\right)$ (as shown in Figure 1 , for the case of two light sources $), \mathbf{l}_{i}(x, y)$ is the light direction that changes at each image point $(x, y)$ as follows:

$$
\mathbf{l}_{i}(x, y)=P_{i}-\mathbf{M}(x, y)
$$

before being normalized. With the triple $(\xi, \eta, \zeta)$ we indicate the real-world coordinates and by $(x, y, z)$ by the perspective or image coordinates.

The setup we take into account exploits the pinhole camera model shown in Figure 1, where the optical and focal planes are respectively as follows:

$$
\zeta=0 \quad \text { and } \quad \zeta=-f
$$

The final model considered in $^{1}$ comes from taking the ratio of a couple of irradiance equations like (1). In the following, we derive more in detail the model according to different surface parametrizations, i.e. different $\mathbf{M}$.

Since both $\mathbf{n}$ and $\mathbf{l}_{i}$ depend directly on $\mathbf{M}$, the way the surface is parametrized plays a fundamental role. The preliminary parametrization we consider is not new in the field of SfS and PS. It is based on projecting the surface on the lower half-sphere of radius $f$. We extend such derivation under the same procedure of projection onto general auxiliary convex surfaces. In other terms, we generalize the parametrization introduced in ${ }^{2}$ (and derived more in detail $\mathrm{in}^{7}$ ) where the lower half-sphere is taken as a specific choice of auxiliary convex function, see Figure 2.

\subsection{Projected parametrization on the half-sphere}

The aim of the perspective model presented $i^{2}$ is to parametrize the surface by using a point projected through the lower half-sphere

$$
\zeta=-\sqrt{f^{2}-\xi^{2}-\eta^{2}}
$$

onto the focal plane, as shown in Figure 2. Let us consider the equations of the segment connecting the optical center $(0,0,0)$ to a generic point in the focal plane $(x, y,-f)$. The parametric and cartesian equations for that segment are

$$
r:\left\{\begin{array}{l}
\xi=x t \\
\eta=y t \\
\zeta=-f t
\end{array} \rightarrow r:\left\{\begin{array}{l}
\xi=-x \frac{\zeta}{f} \\
\eta=-y \frac{\zeta}{f}
\end{array}\right.\right.
$$

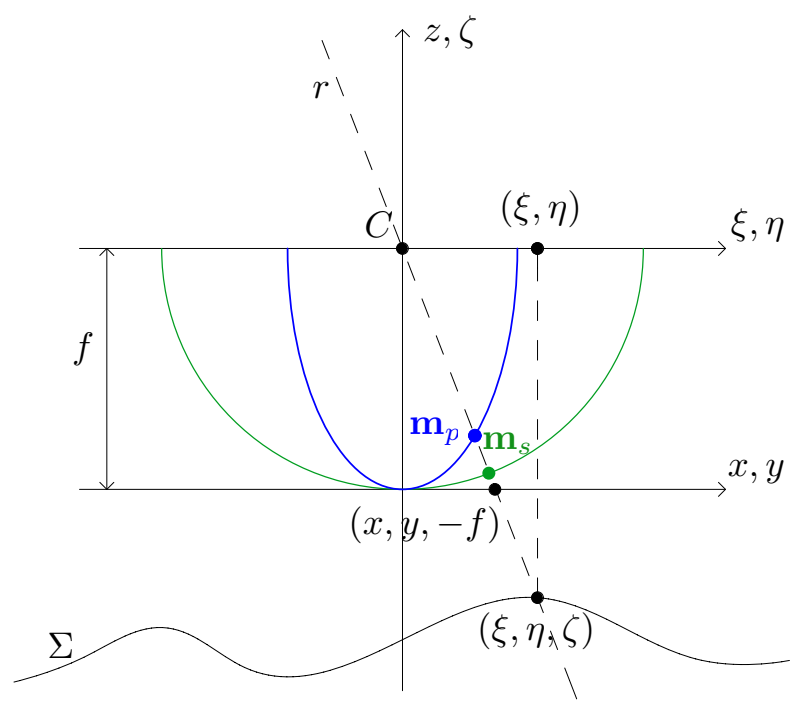

Figure 2. Two different colors for the parametrizations of $\Sigma$ using the lower half sphere $\zeta(\xi, \eta)=-\sqrt{f^{2}-\xi^{2}-\eta^{2}}$ in green and the second by the paraboloid $\zeta(\xi, \eta)=\xi^{2}+\eta^{2}-f$ in blue. 
The point of intersection between this straight line and the lower half-sphere is given by substitution of (6) into (5), that is

$$
\widetilde{\zeta}_{s}(x, y)=-\frac{f^{2}}{\sqrt{f^{2}+x^{2}+y^{2}}}
$$

Substituting (7) into (6) we get the following functions for the projection onto the lower half sphere:

$$
\widetilde{\xi}_{s}(x, y)=\frac{f x}{\sqrt{f^{2}+x^{2}+y^{2}}} \quad \text { and } \quad \widetilde{\eta}_{s}(x, y)=\frac{f y}{\sqrt{f^{2}+x^{2}+y^{2}}} .
$$

The perspective surface is then easily obtained as follows:

$$
\mathbf{M}_{s}(x, y)=\mathbf{m}_{s} u_{s}(x, y)=\left(\widetilde{\xi}_{s}(x, y), \widetilde{\eta}_{s}(x, y), \widetilde{\zeta}_{s}(x, y)\right) u_{s}(x, y)=\left(\frac{f x}{d_{s}(x, y)}, \frac{f y}{d_{s}(x, y)},-\frac{f^{2}}{d_{s}(x, y)}\right) u_{s}(x, y)
$$

where $d_{s}(x, y)=\sqrt{f^{2}+x^{2}+y^{2}}$, and $u_{s}(x, y)$ is the unknown function that parametrizes this perspective representation of the surface.

From here, the computation of the outgoing normal to the surface is straightforward and comes from the cross product of the partial derivatives of (9) with respect to $x$ and $y$ :

$$
\frac{\partial \mathbf{M}_{s}}{\partial x} \times \frac{\partial \mathbf{M}_{s}}{\partial y}=\mathbf{n}_{s}(x, y)=\left(f\left(\frac{\partial u_{s}}{\partial x}-\frac{x u_{s}}{d_{s}^{2}}\right), f\left(\frac{\partial u_{s}}{\partial y}-\frac{y u_{s}}{d_{s}^{2}}\right), \frac{u_{s} f^{2}}{d_{s}^{2}}+x \frac{\partial u_{s}}{\partial x}+y \frac{\partial u_{s}}{\partial y}\right) .
$$

Given this parametrization and according to (3), we derive the following light direction

$$
\mathbf{l}_{i}(x, y)=\left(\xi_{i}-x \frac{f u_{s}}{d_{s}}, \eta_{i}-y \frac{f u_{s}}{d_{s}}, \zeta_{i}+\frac{f^{2} u_{s}}{d_{s}}\right)
$$

and considering the light sources attached to the optical plane (i.e. $\zeta_{i}=0$ ), after some algebraic manipulation of the image ratio $\frac{I_{1}}{I_{2}}$, we get the following quasi-linear PDE:

$$
\begin{aligned}
& \frac{\partial u_{s}}{\partial x} \frac{d_{s}}{u_{s}}\left[a_{1}(x, y) I_{1}(x, y)\left|\mathbf{l}_{1}(x, y)\right| \xi_{2}-a_{2}(x, y) I_{2}(x, y)\left|\mathbf{l}_{2}(x, y)\right| \xi_{1}\right]+ \\
& \frac{\partial u_{s}}{\partial y} \frac{d_{s}}{u_{s}}\left[a_{1}(x, y) I_{1}(x, y)\left|\mathbf{l}_{1}(x, y)\right| \eta_{2}-a_{2}(x, y) I_{2}(x, y)\left|\mathbf{l}_{2}(x, y)\right| \eta_{1}\right]= \\
& f u_{s}\left[a_{2}(x, y) I_{2}(x, y)\left|\mathbf{l}_{2}(x, y)\right|-a_{1}(x, y) I_{1}(x, y)\left|\mathbf{l}_{2}(x, y)\right|\right]+ \\
& \frac{1}{d_{s}}\left[a_{1}(x, y) I_{1}(x, y)\left|\mathbf{l}_{1}(x, y)\right|\left(\xi_{2} x+\eta_{2} y\right)-a_{2}(x, y) I_{2}(x, y)\left|\mathbf{l}_{2}(x, y)\right|\left(\xi_{1} x+\eta_{1} y\right)\right] .
\end{aligned}
$$

\subsection{Projected parametrization on a paraboloid}

According to the previous section, the generalization of a projection on a generic convex surface is easy to extend since it is based on a different choice of the convex function (5). For example, let us consider the projection onto the paraboloid

$$
\zeta=-f+\xi^{2}+\eta^{2}
$$

as shown in Figure 2.

Computing the intersection between the paraboloid and the line (6), we have

$$
\widetilde{\zeta}_{p}(x, y)=-\frac{2 f^{2}}{f+\sqrt{f^{2}+4 f\left(x^{2}+y^{2}\right)}},
$$




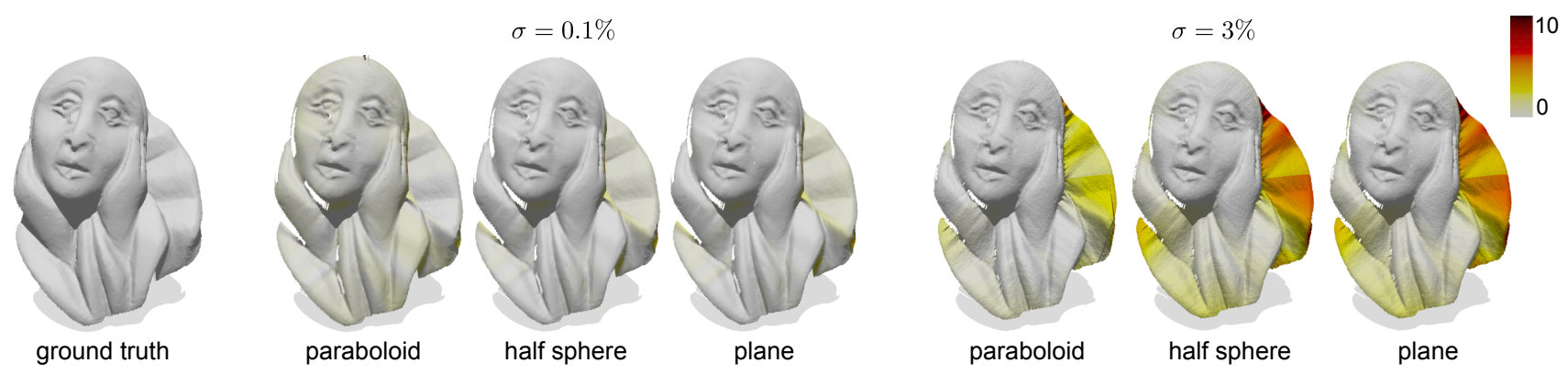

Figure 3. Reconstruction of the "pierrot" shape using different projective models. At low noise levels $(\sigma=0.1 \%)$ all parametrizations behave comparably well, with the parabolic model showing slightly larger MSE than the others. When noise increases $(\sigma=3 \%)$ the situation turns in favor of the former, which yields more stable reconstructions by a good margin. The error scale here is in $\mathrm{mm}^{2}$.

which brings us to the following projection functions:

$$
\widetilde{\xi}_{p}(x, y)=\frac{2 f x}{f+\sqrt{f^{2}+4 f\left(x^{2}+y^{2}\right)}} \quad \text { and } \quad \widetilde{\eta}_{p}(x, y)=\frac{2 f y}{f+\sqrt{f^{2}+4 f\left(x^{2}+y^{2}\right)}} .
$$

Finally, we get surface $\Sigma$ parametrized as follows:

$$
\mathbf{M}_{p}(x, y)=\mathbf{m}_{p} u_{p}(x, y)=\left(\widetilde{\xi}_{p}(x, y), \widetilde{\eta}_{p}(x, y), \widetilde{\zeta}_{p}(x, y)\right) u_{p}(x, y)=\left(\frac{2 f x}{d_{p}(x, y)}, \frac{2 f y}{d_{p}(x, y)},-\frac{2 f^{2}}{d_{p}(x, y)}\right) u_{p}(x, y)
$$

where $d_{p}(x, y)=f+\sqrt{f^{2}+4 f\left(x^{2}+y^{2}\right)}$.

Let us write now the irradiance equation by computing the outgoing normal from the previous formula for the projected surface. The usual procedure is to compute the normal as a perpendicular vector of the tangent plane to the surface. So we start computing the tangent plane as follows:

$$
\frac{\partial \mathbf{M}_{p}}{\partial x}=\left(\left(u_{p}+x \frac{\partial u_{p}}{\partial x}\right) d_{p}-\frac{4 f^{2} u_{p} x^{2}}{d_{p}-f}, y \frac{\partial u_{p}}{\partial x} d_{p}-\frac{4 f^{2} u_{p} x y}{d_{p}-f},-f \frac{\partial u_{p}}{\partial x} d_{p}+\frac{4 f^{2} u_{p} x}{d_{p}-f}\right)
$$

and

$$
\frac{\partial \mathbf{M}_{p}}{\partial y}=\left(x \frac{\partial u_{p}}{\partial y} d_{p}-\frac{4 f^{2} u_{p} x y}{d_{p}-f},\left(u_{p}+y \frac{\partial u_{p}}{\partial y}\right) d_{p}-\frac{4 f^{2} u_{p} y^{2}}{d_{p}-f},-f \frac{\partial u_{p}}{\partial y} d_{p}+\frac{4 f^{2} u_{p} y}{d_{p}-f}\right) .
$$

The (not unit) outgoing normal is easily computed by the cross product

$$
\begin{aligned}
& \frac{\partial \mathbf{M}_{p}}{\partial x} \times \frac{\partial \mathbf{M}_{p}}{\partial y}=\mathbf{n}_{p}(x, y) \\
= & \left(f\left(d_{p} \frac{\partial u_{p}}{\partial x}-\frac{4 f x}{d_{p}-f} u_{p}\right),\right. \\
& f\left(d_{p} \frac{\partial u_{p}}{\partial y}-\frac{4 f y}{d_{p}-f} u_{p}\right), \\
& \left.\frac{d_{p}^{2}\left(u_{p}+x \frac{\partial u_{p}}{\partial x}+y \frac{\partial u_{p}}{\partial y}\right)-d_{p} f\left(u_{p}+x \frac{\partial u_{p}}{\partial x}+y \frac{\partial u_{p}}{\partial y}\right)-4 f u_{p}\left(x^{2}+y^{2}\right)}{d_{p}-f}\right) .
\end{aligned}
$$

Following equation (3), we parametrize the light source as

$$
\mathbf{l}_{i}(x, y)=\left(\xi_{i}-x \frac{2 f u_{p}}{d_{p}}, \eta_{i}-y \frac{2 f u_{p}}{d_{p}}, \zeta_{i}+\frac{2 f^{2} u_{p}}{d_{p}}\right),
$$



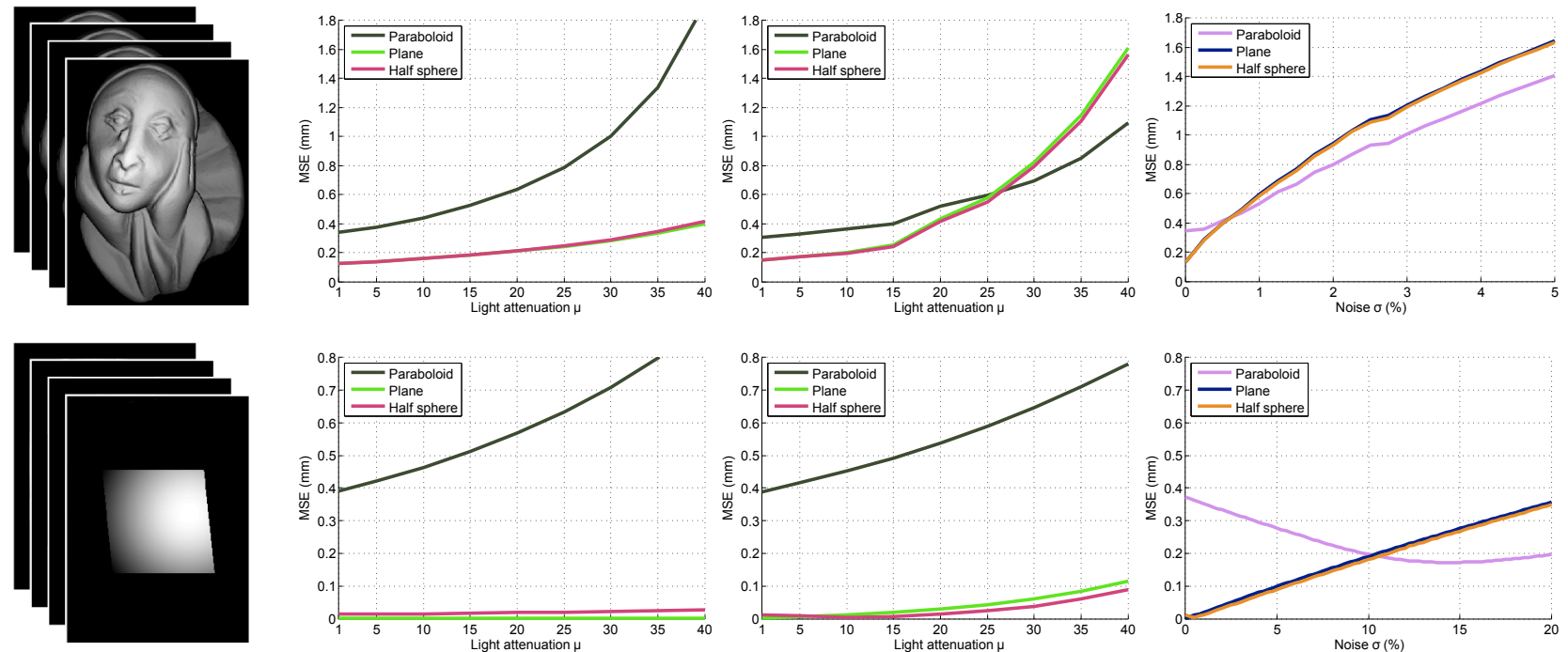

Figure 4. Sensitivity experiments of the three parametrization models under different values of light attenuation $(\mu)$ and pixel noise ( $\sigma$, expressed in percentage of grey levels). The left-most curves show results in the noise-free case, whereas in the middle column we set $\sigma=0.1 \%$. Notice how, in the first row, the parabolic parametrization degrades more gracefully under such moderate noise levels. This behavior is made more evident at fixed $\mu=2$ (right-most plots), where the MSE is even shown to fall down at increasing noise levels. Further explanation of this phenomenon is given in Fig. 5.

and considering two irradiance equations as modeled in (1), namely $I_{1}$ and $I_{2}$, the ratio $\frac{I_{1}}{I_{2}}$ yields the following equation:

$$
\begin{aligned}
& \frac{\partial u_{p}}{\partial x}\left(d_{p} f\left(\frac{a_{2}}{\left|\mathbf{l}_{2}\right|} l_{2}^{1} I_{1}-\frac{a_{1}}{\left|\mathbf{l}_{1}\right|} l_{1}^{1} I_{2}\right)+x d_{p}\left(\frac{a_{2}}{\left|\mathbf{l}_{2}\right|} l_{2}^{3} I_{1}-\frac{a_{1}}{\left|\mathbf{l}_{1}\right|} l_{1}^{3} I_{2}\right)\right)+ \\
& \frac{\partial u_{p}}{\partial y}\left(d_{p} f\left(\frac{a_{2}}{\left|\mathbf{l}_{2}\right|} l_{2}^{2} I_{1}-\frac{a_{1}}{\left|\mathbf{l}_{1}\right|} l_{1}^{2} I_{2}\right)+y d_{p}\left(\frac{a_{2}}{\left|\mathbf{l}_{2}\right|} l_{2}^{3} I_{1}-\frac{a_{1}}{\left|\mathbf{l}_{1}\right|} l_{1}^{3} I_{2}\right)\right) \\
= & \left.\frac{4 f^{2} x}{d_{p}-f} u_{p}\left(\frac{a_{2}}{\left|\mathbf{l}_{2}\right|} l_{2}^{1} I_{1}-\frac{a_{1}}{\left|\mathbf{l}_{1}\right|} l_{1}^{1} I_{2}\right)+\frac{4 f^{2} y}{d_{p}-f} u_{p}\left(\frac{a_{2}}{\left|\mathbf{l}_{2}\right|} l_{2}^{2} I_{1}-\frac{a_{1}}{\left|\mathbf{l}_{1}\right|} l_{1}^{2} I_{2}\right)+\frac{d_{p} f u_{p}+4 f u_{p}\left(x^{2}+y^{2}\right)-d_{p}^{2} u_{p}}{d_{p}-f}\left(\frac{a_{2}}{\left|\mathbf{l}_{2}\right|} l_{2}^{3} I_{1}-\frac{a_{1}}{\left|\mathbf{l}_{1}\right|} l_{1}^{3} I_{2}\right)\right) .
\end{aligned}
$$

For the next section we solved numerically the quasi-linear PDEs we get, comparing the accuracy by showing quantitative results for realistic synthetic cases.

\section{EXPERIMENTAL RESULTS}

We conducted a series of experiments in order to comparatively evaluate the projection models described in the previous sections. For these experiments we employed two synthetic surfaces: a square and perfectly planar object with no features, and the frontal view of a "pierrot" figure (see Fig. 4). Each 3D model was captured with a virtual perspective pinhole camera placed at $\sim 45 \mathrm{~cm}$ from the object, and each object was rescaled to a diameter of $\sim 30 \mathrm{~cm}$. Camera resolution was fixed at $640 \times 480$, and four virtual point light sources were displaced in a radial arrangement on the camera plane.

For comparison purposes, in all experiments we initialized the reconstruction process (an order-one fast marching scheme adapted from ${ }^{1}$ ) with the ground-truth depth value of the central pixel. As error measures, we provide the angular error between the reconstructed and ground-truth normals (degrees), and the point-to-point mean square error (MSE) between the recovered and ground-truth surface $\left(\mathrm{mm}^{2}\right)$.

In Fig. 4 we plot the MSE attained by the three parametrizations under different settings. In this and the following figures, we refer to the three compared projection models as Paraboloid (Sec. 2.2), Half sphere (Sec. 


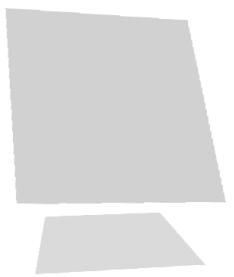

ground truth
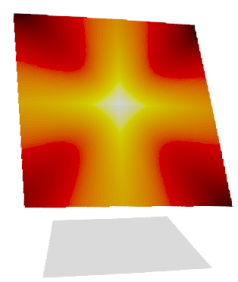

$\sigma=0 \%$
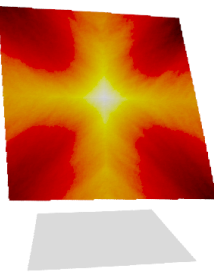

$\sigma=1 \%$

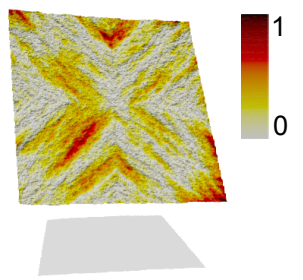

$\sigma=20 \%$

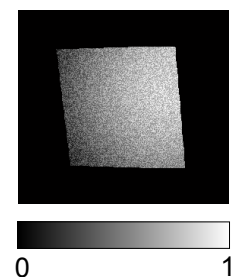

images at $\sigma=20 \%$

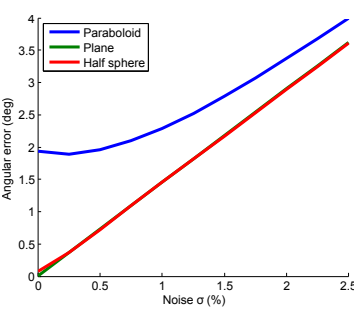

Figure 5. Reconstruction of a planar surface using a parabolic parametrization. The noise-free reconstruction deviates from the true surface in a radial fashion; as more noise is introduced $(\sigma=1-20 \%)$, the reconstruction error distributes more evenly over the whole surface, which regains a flat appearance (with lower MSE). The right-most plot shows the angular error (in degrees) of the reconstructed normals at increasing levels of noise. Notice how this error measure reflects more closely the higher frequency deviations, rather than the global geometry (compare with bottom-right plot of Fig. 4).

2.1), and Plane (straight projection onto the focal plane ${ }^{1}$ ) respectively. The first plot shows MSE curves in a noiseless setting, under increasingly strong light attenuation. The parabolic model seems to be the least stable by a large margin, quickly diverging as $\mu$ increases towards more realistic attenuation values, while the remaining two models remain fairly stable in both the depicted cases. However, as a small amount of pixel noise $(\sigma=0.1 \%)$ is introduced into the source images, the spherical and planar models deviate significantly while the parabolic parametrization gains in resilience (middle column). This is especially evident with the "pierrot" shape, where at $\mu=25$ the relative ranking subverts and the parabolic model starts outperforming the other models (a depiction of this is given in Fig. 3). Evidence of this surprising behavior is further given in the following experiment (right-most curves), where the parabolic model takes the lead as the amount of pixel noise grows into more realistic values. The bottom-right plot additionally shows a counter-intuitive phenomenon: the mean square error seems to decrease for the paraboloid case as noise increases, only to grow again after a certain noise threshold. We visualize this scenario more closely in Fig. 5. For numerical reasons, the reconstruction obtained with the paraboloid model in the noise-free $(\sigma=0 \%)$ case does not approximate the plane well, and rather shows a curved profile. The introduction of noise has a "flattening" effect on the recovered geometry, thus reducing the MSE; as noise is furthermore increased, the global geometry deforms more severely and the reconstruction error grows again.

\section{CONCLUSIONS}

In this work we analyze three different parametrizations of 3D surfaces employed to a modern and realistic PS approach $^{1}$ based on partial differential irradiance equation ratios. After explicitly showing the general derivation for such parametrizations, we implemented them working on synthetic tests. The experimental evaluation reveals an unexpected robustness of the parametrization where the paraboloid was considered as auxiliary convex projection function. This opens to new works mainly focused on choosing the more suitable parametrization for the PS reconstruction process based on sigle step fast marching methods.

\section{Acknowledgments}

Emanuele Rodolà is supported by an Alexander von Humboldt fellowship. The "pierrot" model appears courtesy of the AIM@Shape repository.

\section{REFERENCES}

[1] Mecca, R., Wetzler, A., Bruckstein, A., and Kimmel, R., "Near field photometric stereo with point light sources," SIAM Journal on Imaging Sciences to appear (2014).

[2] Prados, E. and Faugeras, O. D., "Shape from shading: A well-posed problem?," in [CVPR], 2, 870-877, IEEE Computer Society (2005).

[3] Horn, B. K. P. and Brooks, M. J., [Shape from Shading], The MIT Press (1989). 
[4] Woodham, R. J., "Photometric method for determining surface orientation from multiple images," Optical Engineering 19(1), 134-144 (1980).

[5] Durou, J., Falcone, M., and Sagona, M., "Numerical methods for shape from shading: a new survey with benchmarks," Computer Vision and Image Understanding 109(1), 22-43 (2008).

[6] Zhang, R., Tsai, P. S., Cryer, J. E., and Shah, M., "Shape-from-shading: a survey," IEEE Transaction on Pattern Analysis and Machine Intelligence 21(8), 690-706 (1999).

[7] Breuß, M., Cristiani, E., Durou, J., Falcone, M., and Vogel, O., "Perspective shape from shading: Ambiguity analysis and numerical approximations," SIAM Journal on Imaging Sciences 5(1), 311-342 (2012).

[8] Collins, T. and Bartoli, A., "3d reconstruction in laparoscopy with close-range photometric stereo," in [MICCAI], Ayache, N., Delingette, H., Golland, P., and Mori, K., eds., Lecture Notes in Computer Science 7511, 634-642, Springer Berlin Heidelberg (2012).

[9] Crouzil, A., Decombes, X., and Durou, J. D., "A multiresolution approach for shape from shading coupling deterministic and stochastic optimization," IEEE Transactions on Pattern Analysis and Machine Intelligence 25(11), 1416-1421 (2003).

[10] Tankus, A. and Kiryati, N., "Photometric stereo under perspective projection," in [Computer Vision, 2005. ICCV 2005. Tenth IEEE International Conference on], 1, 611-616 Vol. 1 (Oct 2005).

[11] Horn, B. K. P., "Height and gradient from shading," The International Journal of Computer Vision, Winston 5, 37-75 (1990).

[12] Wei, T. and Klette, R., "A new algorithm for gradient field integration," tech. rep., Communication and Information Technology Research Technical Report 103 (2001).

[13] Durou, J. D., Aujol, J.-F., and Courteille, F., "Integrating the normal field of a surface in the presence of discontinuities," in [7th International Conference on Energy Minimization Methods in Computer Vision and Pattern Recognition], 261-273, Springer-Verlag, Berlin, Heidelberg (2009).

[14] Durou, J. D. and Courteille, F., "Integration of a Normal Field without Boundary Condition," in [Proceedings of the First International Workshop on Photometric Analysis For Computer Vision], 8 p., INRIA, Rio de Janeiro, Brésil (2007).

[15] Chandraker, M., Bai, J., and Ramamoorthi, R., "On differential photometric reconstruction for unknown, isotropic brdfs," IEEE Transactions on Pattern Analysis and Machine Intelligence (2012).

[16] Chandraker, M., Bai, J., and Ramamoorthi, R., "A theory of differential photometric stereo for unknown BRDFs," in [IEEE Conference on Computer Vision and Pattern Recognition], 2505-2512 (2011).

[17] Alldrin, N. G. and Kriegman, D. J., "Toward Reconstructing Surfaces With Arbitrary Isotropic Reflectance : A Stratified Photometric Stereo Approach," in [International Conference on Computer Vision], 1-8 (2007).

[18] Mecca, R. and Falcone, M., "Uniqueness and approximation of a photometric shape-from-shading model," SIAM Journal on Imaging Sciences 6(1), 616-659 (2013).

[19] Mecca, R., Tankus, A., Wetzler, A., and Bruckstein, A., "A direct differential approach to photometric stereo with perspective viewing," SIAM Journal on Imaging Sciences 7(2), 579-612 (2014). 